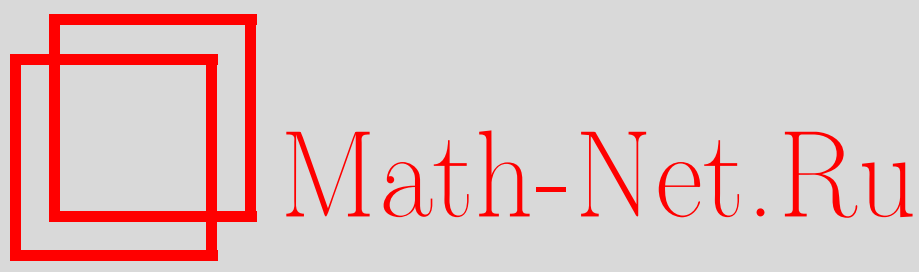

В. В. Щиголев, Примеры бесконечно базируемых T-пространств, Матем. сб., 2000, том 191, номер 3, 143-160

DOI: https://doi.org/10.4213/sm467

Использование Общероссийского математического портала Math-Net.Ru подразумевает, что вы прочитали и согласны с пользовательским соглашением

http: //www.mathnet.ru/rus/agreement

Параметры загрузки:

IP : 35.173 .219 .12

26 апреля 2023 г., 17:46:54 
УДК 512.5

\author{
В.В. Щиголев
}

\title{
Примеры бесконечно базируемых $T$-пространств
}

Работа посвящена построению бесконечно базируемых $T$-пространств надполями конечной характеристики и некоторьми другими кольцами. Приведены примеры $T$-пространств, которые порождены полиномами, зависящими от двух переменных, или полиномами, имеющими по каждой переменной ограниченную степень.

Библиограбоя: 5 названий.

\section{§1. Введение}

В теории ассоциативных PI-колец хорошо известна проблема Шпехта [1] о конечной базируемости любой системы тождеств. В случае нулевой характеристики основного поля эта проблема решена положительно А.Р. Кемером [2]. Им же положительно решена локальная проблема Шпехта в случае, когда основное поле бесконечно [3]. А.Я. Белов анонсировал положительное решение локальной проблемы Шпехта в случае, когда основное поле конечно. А.В. Гришин [4] ввел понятие абстрактного $T$-пространства и доказал конечную базируемость любого $T$-пространства относительно свободной алгебры, содержащей стандартное тождество

$$
\operatorname{St}_{n}\left(x_{1}, \ldots, x_{n}\right)=\sum_{\sigma \in S_{n}} \operatorname{sgn}(\sigma) x_{\sigma(1)} \cdots x_{\sigma(n)}
$$

в случае основного поля характеристики нуль. А.В. Гришин [5] доказал также, что для основного поля характеристики $2 T$-пространство, порожденное многочленами $x_{1}^{2}, x_{1}^{2} x_{2}^{2}, \ldots, x_{1}^{2} \cdots x_{n}^{2}, \ldots$, бесконечно базируемо по модулю тождества $[[x, y], z]=0$.

Напомним, что $T$-пространствами (T-идеалами) алгебры $\Phi\langle X\rangle$, где $\Phi$ - коммутативное кольцо с единицей, а $X$-некоторый счетный алфавит, называются подмодули модуля $\Phi \Phi\langle X\rangle$ (идеалы алгебры $\Phi\langle X\rangle$ ), замкнутые относительно действия всех эндоморфизмов $\Phi\langle X\rangle \rightarrow \Phi\langle X\rangle$. Всюду ниже под выражениями "конечная базируемость" и "бесконечная базируемость" понимается конечная и бесконечная базируемость в смысле $T$-пространств.

В $\S \S 2$ и 3 настоящей работы рассматриваются следующие свободные алгебры с единицей:

$$
F=K\langle X\rangle, \quad M=\mathbb{Z}\langle X\rangle, \quad R(n)=\mathbb{Z}_{n}\langle X\rangle, \quad H\left(p_{1}, \ldots, p_{k}\right)=\mathbb{Q}_{p_{1}, \ldots, p_{k}}\langle X\rangle,
$$

где $X=\left\{x, y, x_{1}, y_{1}, \ldots, x_{n}, y_{n}, \ldots\right\}$ - основной алфавит, $K$ - бесконечное поле характеристики $p \neq 0, \mathbb{Z}$ - кольцо целых чисел, $\mathbb{Z}_{n}$ - кольцо вычетов по модулю $n$, $\mathbb{Q}_{p_{1}, \ldots, p_{k}}=\left\{n / m: n, m \in \mathbb{Z}, n\right.$ не делится ни на одно из чисел $\left.p_{1}, \ldots, p_{k}\right\}-$ локализация поля целых чисел $\mathbb{Q}$ по $p_{1}, \ldots, p_{k}\left(p_{1}, \ldots, p_{k}\right.$ - простые числа).

$$
\text { (C) в. В. Шиголев } 2000
$$


Основным результатом $\S \S 2$ и 3 является следуюшее утверждение:

1) сушествуют однородные полиномы алгебры $F$, зависящие от двух переменных, порождаюшие в $F$ бесконечно базируемое по модулю тождества $[[x, y], z]=0$ $T$-пространство (см. теорему 1).

Далее, существуют следующие естественные эпиморфизмы $\psi_{n}: \mathbb{Z} \rightarrow \mathbb{Z}_{n}$ и $\xi_{k}: \mathbb{Q}_{p} \rightarrow \mathbb{Z}_{p^{k}}$. Кроме того, мы считаем, что $\mathbb{Z} \subset Q_{p_{1}, \ldots, p_{k}}$. Для любого $m \in \mathbb{Z}$ имеем $\psi_{p^{k}}(m)=\xi_{k}(m)$. Эпиморфизмы $\psi_{n}$ и $\xi_{k}$ индуцируют эпиморфизмы $\widehat{\psi}_{n}: M \rightarrow R(n)$ и $\widehat{\xi}_{k}: H(p) \rightarrow R\left(p^{k}\right)$, и мы имеем $M \subset H(p)$.

Используя ту же технику, с помошью которой получен приведенный выше основной результат 1), мы доказываем следующие утверждения:

2 ) сушествует бесконечно базируемое $T$-пространство $L_{1}$ алгебры $M$ такое, что для любых простых чисел $p_{1}, \ldots, p_{k} T$-пространство алгебры $H\left(p_{1}, \ldots, p_{k}\right)$, порожденное $L_{1}$, конечно базируемо, а следовательно, для любого натурального $n$ $T$-пространство $\widehat{\psi}_{n}\left(L_{1}\right)$ алгебры $R(n)$ конечно базируемо (см. теорему 2$)$;

3 ) сушествует $T$-пространство $L_{2}$ алгебры $M$, которое порождает в $H(p)$ бесконечно базируемое $T$-пространство, а для любого натурального $k T$-пространство $\widehat{\xi}_{k}\left(L_{2}\right)$ алгебры $R\left(p^{k}\right)$ конечно базируемо (см. теорему 3$)$.

Заметим, что все упомянутые в утверждениях 1)-3) $T$-пространства порождены полиномами, зависящими от двух переменных.

Представляется интересным открытый пока вопрос о существовании $T$-пространств $L_{1}$ и $L_{2}$, порожденных полиномами, у которых степени по каждой переменной из $X$ ограничены в совокупности и которые удовлетворяют утверждениям 2) и 3$)$.

Далее в $\S 4$ для основного поля характеристики $p>2$ строится пример полиномов степени $p$ или нуль по каждой переменной, которые порождают $T$-пространство, бесконечно базируемое по модулю тождества $[x,[y, z]]=0$ (теорема 4 ). В этом же параграфе показано, что такой пример может быть мономиален, как и в случае, когда характеристика основного поля равна двум (теорема 5).

Параграф 5 посвящен доказательству конечной базируемости системы полиномов $x_{1}^{p}, \ldots, x_{1}^{p} \cdots x_{n}^{p}, \ldots$ при $p>2$, что опровергает предположение А. В. Гришина, сфороулированное в [5].

Автор благодарит В.Н. Латышева, А.В. Гришина и А.Я. Белова за полезные обсуждения.

\section{§ 2. Вычисление однородной компоненты}

Ниже мы используем следующий комбинаторный факт.

Пусть имеется $n$ мест и $n_{i}$ объектов $i$-го класса для любого $i=1, \ldots, k$ и при этом $n_{1}+\cdots+n_{k}=n$. Тогда существует ровно $\frac{n !}{n_{1} ! \cdots n_{k} !}$ способов расставить эти объекты на $n$ мест.

Через $T^{(3)}, T_{\mathbb{Z}}^{(3)}$ и $T_{\mathbb{Z}_{n}}^{(3)}$ обозначим $T$-идеалы алгебр $F, M$ и $R(n)$ соответственно, порожденные многочленом $\left[\left[x_{1}, x_{2}\right], x_{3}\right]$.

Пусть $q_{1}, q_{2}$ - натуральные числа. Через $L^{\left(q_{1}, q_{2}\right)}, L_{\mathbb{Z}}^{\left(q_{1}, q_{2}\right)}$ и $L_{n}^{\left(q_{1}, q_{2}\right)}$ обозначим $T$-пространства алгебр $F, M$ и $R(n)$ соответственно, порожденные многочленами $f_{q_{1}, q_{2}}=x^{q_{1}-1}[x, y] y^{q_{2}-1}$ и $\left[\left[x_{1}, x_{2}\right], x_{3}\right]$. 
Пусть $0 \neq f, g \in \Phi\langle X\rangle$, где $\Phi$ - некоторое коммутативное кольцо. Считаем, что многочлен $g$ является некоторой однородной компонентой многочлена $f$, если многочлен $g$ однородный и у многочлена $f-g$ отсутствуют одночлены $f^{\prime} \neq 0$ такие, что $\operatorname{deg}_{x} g=\operatorname{deg}_{x} f^{\prime}$ для любого $x \in X$.

Выполним замены $x \rightarrow x_{1}+\cdots+x_{k}$ и $y \rightarrow y_{1}+\cdots+y_{l}$. Через $f_{q_{1}, q_{2}}^{\sigma}$ обозначим однородное слагаемое многочлена, получившегося из $f_{q_{1}, q_{2}}$ при этой замене, такое, что $\operatorname{deg}_{x_{i}} f_{q_{1}, q_{2}}^{\sigma}=n_{i} \geqslant 1, i=1, \ldots, k$, и $\operatorname{deg}_{y_{j}} f_{q_{1}, q_{2}}^{\sigma}=m_{j} \geqslant 1, j=1, \ldots, l$, где $\sigma=\left(n_{1}, \ldots, n_{k}, m_{1}, \ldots, m_{l}\right)$.

Ясно, что должны выполнятся соотношения

$$
\sum_{i=1}^{k} n_{i}=q_{1}, \quad \sum_{j=1}^{l} m_{j}=q_{2}
$$

Положим далее $M_{0}=\mathbb{Z}\langle x, y\rangle$ и $M_{k, l}=\mathbb{Z}\left\langle x_{1}, \ldots, x_{k}, y_{1}, \ldots, y_{l}\right\rangle$. Справедливы включения $M_{0} \subset M$ и $M_{k, l} \subset M$.

Далее выполним замену $x_{i} \rightarrow u_{i}$ и $y_{j} \rightarrow v_{j}$, где $u_{i}, v_{j}-$ слова алгебры $M_{0}$ и $\operatorname{deg}_{x} u_{i}=a_{i}, \operatorname{deg}_{y} u_{i}=b_{i}, \operatorname{deg}_{x} v_{j}=c_{j}, \operatorname{deg}_{y} v_{j}=d_{j}$. Гомоморфизм алгебры $M_{k, l}$ в алгебру $M$, определяемый этой заменой, обозначим через $\eta$.

Рассмотрим однородньй многочлен $\eta\left(f_{q_{1}, q_{2}}^{\sigma}\right)$. Приведем его по модулю $T_{\mathbb{Z}}^{(3)} \mathrm{k}$ виду $\gamma[x, y] x^{r_{1}-1} y^{r_{2}-1}, \gamma \in \mathbb{Z}$, и вычислим $\gamma$.

Заметим, что $\left[x, x_{1}\right]\left[x, x_{2}\right] \in T_{\mathbb{Z}}^{(3)}$. Отсюда следует, что $\left[h_{1}, h_{2}\right]\left[h_{3}, h_{4}\right] \in T_{\mathbb{Z}}^{(3)}$, если $h_{1}, h_{2}, h_{3}, h_{4} \in M_{0}$.

Имеем

$$
\begin{aligned}
h_{1}\left[h_{2}\left[h_{3}, h_{4}\right] h_{5}, h_{6}\right] h_{7} & =h_{1}\left[\left[h_{3}, h_{4}\right] h_{5} h_{2}, h_{6}\right] h_{7} \\
& =h_{1}\left[h_{3}, h_{4}\right]\left[h_{5} h_{2}, h_{6}\right] h_{7}\left(\bmod T_{\mathbb{Z}}^{(3)}\right) .
\end{aligned}
$$

Отсюда вытекает, что справедлива

Лемма 1. Пусть $h_{1}, \ldots, h_{7} \in M_{0}$. Тогда

$$
h_{1}\left[h_{2}\left[h_{3}, h_{4}\right] h_{5}, h_{6}\right] h_{7}=0 \quad\left(\bmod T_{\mathbb{Z}}^{(3)}\right) .
$$

ДокАЗАТЕЛЬСтво. Имеем $h_{1}\left[h_{3}, h_{4}\right]\left[h_{5} h_{2}, h_{6}\right] h_{7}=0\left(\bmod T_{\mathbb{Z}}^{(3)}\right)$. Лемма доказана.

Тогда получаем соотношения

$$
\begin{aligned}
{\left[u_{i}, v_{j}\right] } & =\left[x^{a_{i}} y^{b_{i}}, x^{c_{j}} y^{d_{j}}\right]=\left[x^{a_{i}}, y^{d_{j}}\right] x^{c_{j}} y^{b_{i}}+\left[y^{b_{i}}, x^{c_{j}}\right] x^{a_{i}} y^{d_{j}} \\
& =a_{i} d_{j}[x, y] x^{c_{j}+a_{i}-1} y^{b_{i}+d_{j}-1}+b_{i} c_{j}[y, x] x^{a_{i}+c_{j}-1} y^{d_{j}+b_{i}-1} \\
& =\left(a_{i} d_{j}-b_{i} c_{j}\right)[x, y] x^{a_{i}+c_{j}-1} y^{b_{i}+d_{j}-1}\left(\bmod T_{\mathbb{Z}}^{(3)}\right) .
\end{aligned}
$$

Теперь посмотрим, сколько раз выражения $w_{i}^{\prime}\left[x_{i}, y_{j}\right] w_{j}^{\prime \prime}$, где $w_{i}^{\prime}$ и $w_{j}^{\prime \prime}-$ слова, встречаются в $f_{q_{1}, q_{2}}^{\sigma}$. Для расстановки переменных $x_{1}, \ldots, x_{k}$ на те места, где в $f_{q_{1}, q_{2}}^{\sigma}$ стоит $x^{q_{1}-1}$, имеется $q_{1}-1$ мест. При этом $x_{i}$ должно входить $n_{i}-1$ раз, а $x_{i^{\prime}}$ должно входить $n_{i^{\prime}}$ раз при $i^{\prime} \neq i$. Используя формулы комбинаторики, получаем 
$\frac{\left(q_{1}-1\right) !}{n_{1} ! \cdots n_{i-1} !\left(n_{i}-1\right) ! n_{i+1} ! \cdots n_{k} !}=n_{i} \frac{\left(q_{1}-1\right) !}{n_{1} ! \cdots n_{k} !}$ вариантов расстановки. Аналогично, для расстановки переменных $y_{1}, \ldots, y_{l}$ имеется $m_{j} \frac{\left(q_{2}-1\right) !}{m_{1} ! \cdots m_{l} !}$ вариантов. Обшее количество интересуюших нас выражений получается равным $n_{i} m_{j} E$, где $E=\frac{\left(q_{1}-1\right) !}{\left(q_{2}-1\right) ! n_{1} ! \cdots n_{k} ! m_{1} ! \cdots m_{l} !}$. Заметим, что $E$ может быть нецелым.

Таким образом, слагаемое $\left(a_{i} d_{j}-b_{i} c_{j}\right)[x, y] x^{r_{1}-1} y^{r_{2}-1}$ надо взять $n_{i} m_{j} E$ раз. Отсюда

$$
\begin{aligned}
\gamma & =\sum_{i=1}^{k} \sum_{j=1}^{l}\left(a_{i} d_{j}-b_{i} c_{j}\right) n_{i} m_{j} E \\
& =\sum_{i=1}^{k} \sum_{j=1}^{l}\left(\left(n_{i} a_{i}\right)\left(m_{j} d_{j}\right)-\left(n_{i} b_{i}\right)\left(m_{j} c_{j}\right)\right) E=(A D-B C) E
\end{aligned}
$$

где $A=\sum_{i=1}^{k} n_{i} a_{i}, B=\sum_{i=1}^{k} n_{i} b_{i}, C=\sum_{j=1}^{l} m_{j} c_{j}$ и $D=\sum_{j=1}^{l} m_{j} d_{j}$.

Вычислим теперь показатели степени $r_{1}, r_{2}$. Так как переменная $x_{i}$ входит в каждый моном $f_{q_{1}, q_{2}}^{\sigma} n_{i}$ раз, а вместо нее подставляется слово $u_{i}$ степени $a_{i}$ по $x$ и $b_{i}$ по $y$, то степень по $x$ оказьвается равной $n_{i} a_{i}$, а по $y-n_{i} b_{i}$. Аналогично, переменная $y_{j}$ дает вклад $m_{j} c_{j}$ по $x$ и $m_{j} d_{j}$ по $y$. Суммируя по $i=1, \ldots, k$ и $j=1, \ldots, l$, получаем

$$
r_{1}=\sum_{i=1}^{k} n_{i} a_{i}+\sum_{j=1}^{l} m_{j} c_{j}=A+C, \quad r_{2}=\sum_{i=1}^{k} n_{i} b_{i}+\sum_{j=1}^{l} m_{j} d_{j}=B+D .
$$

Тогда справедливо соотношение

$$
\gamma=(A D-B C) E=\left(\left(r_{1}-C\right)\left(r_{2}-B\right)-B C\right) E=\left(r_{1} r_{2}-r_{1} B-r_{2} C\right) E .
$$

Для дальнейшего нам будет важно знать величину $\gamma$ в случае, когда $r_{1}=r_{2}=$ $q_{0} q_{1}=q_{0} q_{2} \geqslant 1$. Положим $r=r_{1}=r_{2}$ и $q=q_{1}=q_{2}$.

Формула (2) теперь переписывается в виде

$$
\gamma=\left(r^{2}-r(B-C)\right) E .
$$

Имеем

$$
\operatorname{Er}^{2}=q_{0}^{2} \cdot \frac{q !}{n_{1} ! \cdots n_{k} !} \cdot \frac{q !}{m_{1} ! \cdots m_{l} !}
$$

Далее получаем

$$
\begin{aligned}
r B E & =q_{0} \sum_{i=1}^{k} b_{i} n_{i} \frac{(q-1) !}{n_{1} ! \cdots n_{k} !} \cdot \frac{q !}{m_{1} ! \cdots m_{l} !} \\
& =q_{0} \sum_{i=1}^{k} b_{i} \frac{(q-1) !}{n_{1} ! \cdots n_{i-1} !\left(n_{i}-1\right) ! n_{i+1} ! \cdots n_{k} !} \cdot \frac{q !}{m_{1} ! \cdots m_{l} !} .
\end{aligned}
$$

Аналогично имеем

$$
r C E=q_{0} \sum_{j=1}^{l} c_{j} \frac{q !}{n_{1} ! \cdots n_{k} !} \cdot \frac{(q-1) !}{m_{1} ! \cdots m_{j-1} !\left(m_{j}-1\right) ! m_{j+1} ! \cdots m_{l} !} .
$$

Из приведенных выше равенств и формул комбинаторики видно, что $\gamma$ делится на $q_{0}$. Таким образом, справедлива 
Лемма 2. Если $r=q_{0} q$, то $\gamma$ делится на $q_{0}$.

Приведем теперь еще один факт о многочленах $f_{q_{1}, q_{2}}$. Пусть $V^{\left(q_{1}, q_{2}\right)}-$ свободньй $\mathbb{Z}$-модуль, порожденньй всеми словами алгебры $M_{0}$ степени $q_{1}$ по $x$ и $q_{2}$ по $y$. Определим гомоморфизм $\varphi$ модуля ${ }_{\mathbb{Z}} V^{\left(q_{1}, q_{2}\right)}$ в модуль $\mathbb{Z} \mathbb{Z}$ на словах из $M_{0}$ следующим образом:

$$
\varphi\left(x^{a_{1}} y^{b_{1}} \cdots x^{a_{n}} y^{b_{n}}\right)=\sum_{i<j} b_{i} a_{j} .
$$

Другими словами, $\varphi(u)$, где $u$ - слово, есть число перестановок $y x \rightarrow x y$, необходимых для приведения $u$ к виду $x^{q_{1}} y^{q_{2}}$. Далее слова из $M_{0}$, в которых все $x$ предшествуют всем $y$, называем правильнымми.

Справедлива

ЛЕмма 3. Eсли $h_{1}, \ldots, h_{5} \in M_{0}, \operatorname{deg}_{x} h_{1} \cdots h_{5}=q_{1} u \operatorname{deg}_{y} h_{1} \cdots h_{5}=q_{1}, m o$

$$
\varphi\left(h_{1}\left[\left[h_{2}, h_{3}\right], h_{4}\right] h_{5}\right)=0 .
$$

ДокАЗАтельство. Очевидно, можно считать, что $h_{1}, h_{2}, h_{3}, h_{4}, h_{5}$ - слова. Пусть $g=u_{1}\left[\left[u_{2}, u_{3}\right], u_{4}\right] u_{5}$, где $u_{1}, \ldots, u_{5}-$ слова из $M_{0}$. Обозначим через $r_{i}$ количество перестановок $y x \rightarrow x y$, нужных для приведения $u_{i}$ к правильному виду, где $i=1, \ldots, 5$. Пусть $\operatorname{deg}_{x} u_{i}=a_{i}$ и $\operatorname{deg}_{y} u_{i}=b_{i}, i=1, \ldots, 5$.

Тогда

$$
\begin{aligned}
& \varphi\left(u_{1} u_{\sigma(2)} u_{\sigma(3)} u_{\sigma(4)} u_{5}\right)=a_{5}\left(b_{2}+b_{3}+b_{4}\right)+b_{1}\left(a_{2}+a_{3}+a_{4}\right)+a_{5} b_{1} \\
& +r_{1}+\cdots+r_{5}+\varphi\left(x^{a_{1}+a_{5}} x^{a_{\sigma(2)}} y^{b_{\sigma(2)}} x^{a_{\sigma(3)}} y^{b_{\sigma(3)}} x^{a_{\sigma(4)}} y^{b_{\sigma(4)}} y^{b_{1}+b_{5}}\right),
\end{aligned}
$$

где $\sigma$ - взаимно однозначное отображение множества $\{2,3,4\}$ на себя.

Поэтому $\varphi(g)=\varphi\left(x^{a_{1}+a_{5}}\left[\left[x^{a_{2}} y^{b_{2}}, x^{a_{3}} y^{b_{3}}\right], x^{a_{4}} y^{b_{4}}\right] y^{b_{1}+b_{5}}\right)$.

В соответствии с формулой (4)

$$
\begin{aligned}
& \varphi\left(x^{a_{1}+a_{5}} x^{a_{2}} y^{b_{2}} x^{a_{3}} y^{b_{3}} x^{a_{4}} y^{b_{4}} y^{b_{1}+b_{5}}\right)=b_{3} a_{4}+b_{2}\left(a_{3}+a_{4}\right) \\
& \varphi\left(x^{a_{1}+a_{5}} x^{a_{3}} y^{b_{3}} x^{a_{2}} y^{b_{2}} x^{a_{4}} y^{b_{4}} y^{b_{1}+b_{5}}\right)=b_{2} a_{4}+b_{3}\left(a_{2}+a_{4}\right) \\
& \varphi\left(x^{a_{1}+a_{5}} x^{a_{4}} y^{b_{4}} x^{a_{2}} y^{b_{2}} x^{a_{3}} y^{b_{3}} y^{b_{1}+b_{5}}\right)=b_{2} a_{3}+b_{4}\left(a_{2}+a_{3}\right) \\
& \varphi\left(x^{a_{1}+a_{5}} x^{a_{4}} y^{b_{4}} x^{a_{3}} y^{b_{3}} x^{a_{2}} y^{b_{2}} y^{b_{1}+b_{5}}\right)=b_{3} a_{2}+b_{4}\left(a_{3}+a_{2}\right) .
\end{aligned}
$$

Отсюда легко видеть, что $\varphi(g)=0$. Лемма доказана.

Заметим теперь, что

$$
\varphi\left(f_{q_{1}, q_{2}}\right)=\varphi\left(x^{q_{1}-1}[x, y] y^{q_{2}-1}\right)=\varphi\left(x^{q_{1}} y^{q_{2}}\right)-\varphi\left(x^{q_{1}-1} y x y^{q_{2}-1}\right)=-1 .
$$

\section{§3. Построение $T$-пространств}

Считаем далее, что $\mathbb{Z}_{p} \subset K$. Определим отображения (проекции) $\mu^{\left(q_{1}, q_{2}\right)}$ : $F \rightarrow F, \mu_{n}^{\left(q_{1}, q_{2}\right)}: R(n) \rightarrow R(n)$ следующим образом:

$$
\mu(u)=\left\{\begin{array}{l}
u, \text { если } u \text { зависит только от } x \text { и } y \text { и } \operatorname{deg}_{x} u=q_{1}, \operatorname{deg}_{y} u=q_{2}, \\
0 \text { иначе, }
\end{array}\right.
$$

где $u$ - слово из $\langle X\rangle$, а $\mu$ - любой из символов $\mu^{\left(q_{1}, q_{2}\right)}$ или $\mu_{n}^{\left(q_{1}, q_{2}\right)}$.

Лемма 4. Пусть $t>s$. Тогда $\mu^{\left(p^{t}, p^{t}\right)}\left(L^{\left(p^{s}, p^{s}\right)}\right) \subset T^{(3)}$. 
ДокАЗАТЕЛЬство. Очевидно, можно считать, что любой элемент из $\mu^{\left(p^{t}, p^{t}\right)}\left(L^{\left(p^{s}, p^{s}\right)}\right)$ является линейной комбинацией над $K$ многочленов $\widehat{\psi}_{p} \eta\left(f_{p^{s}, p^{s}}^{\sigma}\right)$ для различных допустимых $\sigma$ и $\eta$, т.е. таких $\sigma$ и $\eta$, что степени многочлена $\eta\left(f_{p^{s}, p^{s}}^{\sigma}\right)$ по всем переменным совпадают с соответствуюшими степенями многочлена $f_{p^{t}, p^{t}}$. Если в лемме 2 положить $r=p^{t}, q=p^{s}, q_{0}=p^{t-s}$, то получим, что $\widehat{\psi}_{p} \eta\left(f_{p^{s}, p^{s}}^{\sigma}\right) \in T^{(3)}$. Лемма доказана.

ТЕОРемА 1. T-пространство алгебры $F$, порожсденное полиномами

$$
\left\{\widehat{\psi}_{p}\left(f_{p^{s}, p^{s}}\right): s \in \mathbb{N}\right\}
$$

является бесконечно базируемым по модулю тождества $[[x, y], z]=0$.

ДокАЗАтЕльство. По лемме 2 получаем $\widehat{\psi}_{p}\left(f_{p^{s}, p^{s}}\right) \notin T^{(3)}$. Отсюда и из леммы 4 следует утверждение теоремы.

Положим теперь $m(s)=\tau(1) \cdots \tau(s)$, где $\tau(i)$ есть $i$-е простое число. Заметим, что $L_{n}^{\left(q_{1}, q_{2}\right)}=\widehat{\psi}_{n}\left(L_{\mathbb{Z}}^{\left(q_{1}, q_{2}\right)}\right)$.

Лемма 5. Пусть $t>s$. Тогда $\mu_{\tau(s+1)}^{(m(t), m(t))}\left(L_{\tau(s+1)}^{(m(s), m(s))}\right) \subset T_{\mathbb{Z}_{\tau(s+1)}^{(3)}}$.

Доказательство получается применением леммы 2 при $r=m(t), q=m(s)$ и $q_{0}=\tau(s+1) \cdots \tau(t)$.

Теорема 2. T-пространство $L_{1}$ алгебры $M$, порохсденое многочленами $\left\{f_{m(s), m(s)}: s \in \mathbb{N}\right\}$ и T-идеалом $T_{\mathbb{Z}}^{(3)}$, бесконечно базируемо. Но для любих простых чисел $p_{1}, \ldots, p_{k}$ T-пространство алгебры $H\left(p_{1}, \ldots, p_{k}\right)$, порохсденное $L_{1}$, конечно базируемо.

ДокАЗАТЕЛьство. Первое утверждение теоремы следует из лемм 5 и 2.

Далее, при $t>s$ имеем

$$
\begin{aligned}
& \left(x^{\tau(s+1) \cdots \tau(t)}\right)^{m(s)-1}\left[x^{\tau(s+1) \cdots \tau(t)}, y^{\tau(s+1) \cdots \tau(t)}\right]\left(y^{\tau(s+1) \cdots \tau(t)}\right)^{m(s)-1} \\
& =(\tau(s+1) \cdots \tau(t))^{2} x^{m(t)-1}[x, y] y^{m(t)-1}\left(\bmod T_{\mathbb{Z}}^{(3)}\right)
\end{aligned}
$$

Пусть теперь $p_{1}<\cdots<p_{k}=\tau(s)$ - простые числа. Тогда число $(\tau(s+1)$ $\cdots \tau(t))^{2}$ не делится ни на одно из чисел $p_{1}, \ldots, p_{k}$ и полиномы $x_{1}\left[\left[x_{2}, x_{3}\right], x_{4}\right] x_{5}$, $f_{m(1), m(1)}, \ldots, f_{m(s), m(s)}$ образуют базис $T$-пространства алгебры $H\left(p_{1}, \ldots, p_{k}\right)$, порожденного $L_{1}$. Теорема доказана.

Пусть $g_{s}=p^{s} f_{p^{s^{2}}, p^{s^{2}}}$ и $L_{2}-T$-пространство алгебры $M$, порожденное многочленами $\left\{g_{s}: s \in \mathbb{N}\right\}$ и $T$-идеалом $T_{\mathbb{Z}}^{(3)}$. Легко видеть, что $T$-пространство $\widehat{\xi}_{k}\left(L_{2}\right)$ конечно базируемо при любом $k$, так как лишш конечное число многочленов $\left\{\widehat{\xi}_{k}\left(g_{s}\right): s \in \mathbb{N}\right\}$ отлично от нуля.

Пусть $t^{2}-s^{2}>t$. Если в лемме 2 положить $r=p^{t^{2}}, q=p^{s^{2}}$ и $q_{0}=p^{t^{2}-s^{2}}$, то получаем следующее утверждение.

ЛЕмма 6. Имеет место включение

$$
\mu_{p^{t+1}}^{\left(p^{t^{2}}, p^{t^{2}}\right)} L_{p^{s+1}}^{\left(p^{t^{2}}, p^{t^{2}}\right)} \subset T_{p^{s+1}}^{(3)} .
$$

С другой стороны, по лемме 2 имеем $g_{t} \notin T_{\mathbb{Z}_{p^{t+1}}^{(3)}}$.

Таким образом, справедлива 
Теорема 3. T-пространство алгебры $H(p)$, порожденное $L_{2}$, бесконечно базируемо. Но для любого $k$ T-пространство $\widehat{\xi}_{k}\left(L_{2}\right)$ алгебры $R\left(p^{k}\right)$ конечно базируемо.

\section{$\S 4$. Случай ограниченных степеней}

В этом параграфе мы будем использовать обозначения, независимые от $\S \S 2$ и 3 . Пусть $K$ - бесконечное поле характеристики $p \neq 0, F=K\left\langle x_{1}, \ldots, x_{n}, \ldots\right\rangle, \widetilde{F}=$ $K\left\langle x_{1}, y_{1}, \ldots, x_{n}, y_{n}, \ldots\right\rangle$ - алгебры с единицей, а $T^{(3)}$ и $\widetilde{T}^{(3)}$ - идеалы алгебр $F$ и $\widetilde{F}$ соответственно, порожденные полиномом $\left[\left[x_{1}, x_{2}\right], x_{3}\right]$. Считаем, что $F \subset \widetilde{F}$. Везде ниже выражение $z \in f$, где $f$ - однородный полином, означает, что $\operatorname{deg}_{z} f>0$. Пусть также $S_{p}$ обозначает симметрическую группу степени $p$.

Положим $\varphi_{m}=x_{1}^{p-1}\left[x_{1}, x_{2}\right] x_{2}^{p-1} \cdots x_{m-1}^{p-1}\left[x_{m-1}, x_{m}\right] x_{m}^{p-1}$ для четного $m$. При этом считаем, что $\varphi_{0}=1$. Для любых $f_{1}, \ldots, f_{p}, f_{p+1}, g_{1}, \ldots, g_{p} \in \widetilde{F}$ пусть

$$
\begin{aligned}
B\left(f_{1}, \ldots, f_{p}, g_{p}, \ldots, g_{1}\right) & =\sum_{\sigma, \tau \in S_{p}} f_{\sigma(1)} \cdots f_{\sigma(p-1)}\left[f_{\sigma(p)}, g_{\tau(p)}\right] g_{\tau(p-1)} \cdots g_{\tau(1)} \\
D\left(f_{1}, \ldots, f_{p}, f_{p+1}\right) & =\sum_{\sigma \in S_{p}} f_{\sigma(1)} \cdots f_{\sigma(p-1)}\left[f_{\sigma(p)}, f_{p+1}\right] f_{p+1}^{p-1}
\end{aligned}
$$

Здесь и далее выражение $\prod_{i=1}^{n} f_{i}$ интерпретируется как $f_{1} \cdots f_{n}$ (некоммутативное умножение).

Имеет место

Лемма 7. T-пространство $L_{m}$ алгебры $F$, порохсденное многочленом $\varphi_{m}$ и Т-идеалом $T^{(3)}$, есть линейное пространство над $K$, натянутое на $T$-идеал $T^{(3)}$ и на полиномы вида

$$
\begin{aligned}
& \psi_{2 q}\left(v_{1,1}, \ldots, v_{1, p}, \ldots, v_{2 q, 1}, \ldots, v_{2 q, p}, v_{2 q+1}, \ldots, v_{m}\right) \\
& =\prod_{i=1}^{q} B\left(v_{2 i-1,1}, \ldots, v_{2 i-1, p}, v_{2 i, p}, \ldots, v_{2 i, 1}\right) \prod_{j=q+1}^{m / 2} v_{2 j-1}^{p-1}\left[v_{2 j-1}, v_{2 j}\right] v_{2 j}^{p-1} \\
& \psi_{2 q-1}\left(v_{1,1}, \ldots, v_{1, p}, \ldots, v_{2 q-1,1}, \ldots, v_{2 q-1, p}, v_{2 q}, \ldots, v_{m}\right) \\
& =\prod_{i=1}^{q-1} B\left(v_{2 i-1,1}, \ldots, v_{2 i-1, p}, v_{2 i, p}, \ldots, v_{2 i, 1}\right) \\
& \quad \times D\left(v_{2 q-1,1}, \ldots, v_{2 q-1, p}, v_{2 q}\right) \prod_{j=q+1}^{m / 2} v_{2 j-1}^{p-1}\left[v_{2 j-1}, v_{2 j}\right] v_{2 j}^{p-1}
\end{aligned}
$$

əде $v_{i, j}$ и $v_{k}$ - некоторые слова из $F$.

ДоказАТЕЛьство. Имеем

$$
\begin{aligned}
& x_{1}^{p-1}\left[x_{1}, x_{3}\right] x_{3}^{p-1} x_{2}^{p-1}\left[x_{2}, x_{4}\right] x_{4}^{p-1} \\
& \quad=-x_{1}^{p-1}\left[x_{1}, x_{2}\right] x_{2}^{p-1} x_{3}^{p-1}\left[x_{3}, x_{4}\right] x_{4}^{p-1}\left(\bmod T^{(3)}\right) .
\end{aligned}
$$


Линеаризуя это выражение (в силу бесконечности поля $K$ ) по переменным $x_{1}$ и $x_{2}$, получаем

$$
\begin{aligned}
& D\left(f_{1}, \ldots, f_{p}, f_{p+1}\right) D\left(g_{1}, \ldots, g_{p}, g_{p+1}\right) \\
& \quad=-B\left(f_{1}, \ldots, f_{p}, g_{p}, \ldots, g_{1}\right) f_{p+1}^{p-1}\left[f_{p+1}, g_{p+1}\right] g_{p+1}^{p-1}\left(\bmod T^{(3)}\right) .
\end{aligned}
$$

Легко видеть, что $T$-пространство $L_{m}$ есть линейное пространство над $K$, натянутое на $T$-идеал $T^{(3)}$ и некоторые произведения $A_{1} \cdots A_{m}$, где $A_{i}$ есть либо $D\left(u_{1}, \ldots, u_{p}, u_{p+1}\right)$, либо $B\left(u_{1}, \ldots, u_{p}, v_{p}, \ldots, v_{1}\right)$, либо $v_{1}^{p-1}\left[v_{1}, v_{2}\right] v_{2}^{p-1}$, а $u_{1}, \ldots, u_{p+1}, v_{1}, \ldots, v_{p+1}$ - некоторые слова алгебры $F$. Используя соотношение (5), мы избавляемся от всех $D\left(u_{1}, \ldots, u_{p}, u_{p+1}\right)$, кроме, может быть, одного.

Далее имеем

$$
\begin{aligned}
& {\left[x_{1}^{p-1}\left[x_{1}, x_{2}\right] x_{2}^{p-1}, x_{3}\right]=\left[x_{1}^{p-1} x_{2}^{p-1}, x_{3}\right]\left[x_{1}, x_{2}\right]} \\
& =(p-1)\left[x_{1}, x_{3}\right]\left[x_{1}, x_{2}\right] x_{1}^{p-2} x_{2}^{p-1} \\
& \quad+(p-1)\left[x_{2}, x_{3}\right]\left[x_{1}, x_{2}\right] x_{1}^{p-1} x_{2}^{p-2}=0\left(\bmod T^{(3)}\right) .
\end{aligned}
$$

Линеаризуя это выражение по переменной $x_{1}$, получаем

$$
\left[D\left(f_{1}, \ldots, f_{p}, f_{p+1}\right), g\right]=0 \quad\left(\bmod \widetilde{T}^{(3)}\right) .
$$

Линеаризуя то же выражение по переменньм $x_{1}$ и $x_{2}$, получаем

$$
\left[B\left(f_{1}, \ldots, f_{p}, g_{p}, \ldots, g_{1}\right), g_{p+1}\right]=0 \quad\left(\bmod \widetilde{T}^{(3)}\right) .
$$

Теперь, используя формулы (6) и (7), получаем утверждение леммы.

Однородньй полином $f \in \widetilde{F}$ назовем $p$-полнылм, если для любой переменной $z$ из $\widetilde{F}$ выполнено равенство $\operatorname{deg}_{z} f=0(\bmod p)$.

Основной технической задачей является доказательство равенства нулю по модулю $T$-идеала $T^{(3)}$ полиномов

$$
\psi_{2 q}\left(v_{1,1}, \ldots, v_{2 q, p}, v_{2 q+1}, \ldots, v_{m}\right), \quad \psi_{2 q-1}\left(v_{1,1}, \ldots, v_{2 q-1, p}, v_{2 q}, \ldots, v_{m}\right)
$$

в случае, когда $p$-полны полиномы $\prod_{i=1}^{2 q} \prod_{j=1}^{p} v_{i, j}, \prod_{i=1}^{2 q-1} \prod_{j=1}^{p} v_{i, j}$ и $q \geqslant 1$.

Для приведения задачи к более симметричному виду сначала докажем равенство нулю по модулю $\widetilde{T}^{(3)}$ полиномов

$$
\begin{aligned}
& \psi_{2 q}\left(v_{1,1}, \ldots, v_{2 q, p}, v_{2 q+1}, \ldots, v_{m}\right) y_{1}^{p-1}\left[y_{1}, y_{2}\right] y_{2}^{p-1} \cdots y_{2 q-1}^{p-1}\left[y_{2 q-1}, y_{2 q}\right] y_{2 q}^{p-1} \\
& \psi_{2 q-1}\left(v_{1,1}, \ldots, v_{2 q-1, p}, v_{2 q}, \ldots, v_{m}\right) y_{1}^{p-1}\left[y_{1}, y_{2}\right] y_{2}^{p-1} \cdots y_{2 q-1}^{p-1}\left[y_{2 q-1}, y_{2 q}\right] y_{2 q}^{p-1}
\end{aligned}
$$

при выполнении условий на $v_{i, j}$, сфформулированных выше.

Аналогично тому, как было доказано соотношение (5), получаем

$$
\begin{aligned}
& D\left(f_{1}, \ldots, f_{p}, f_{p+1}\right) g_{1}^{p-1}\left[g_{1}, g_{2}\right] g_{2}^{p-1} \\
& \quad=D\left(f_{1}, \ldots, f_{p}, g_{1}\right) f_{p+1}^{p-1}\left[f_{p+1}, g_{2}\right] g_{2}^{p-1}\left(\bmod \widetilde{T}^{(3)}\right) .
\end{aligned}
$$

Таким образом, в силу соотношений (5) и (10) для доказательства равенства нулю по модулю $T$-идеала $\widetilde{T}^{(3)}$ полиномов $(8)$ и $(9)$ достаточно доказать следующую лемму. 
ЛЕмма 8. Пусть полином $\prod_{i=1}^{r} \prod_{j=1}^{p} v_{i, j}$ p-полный. Тогда справедливо соотношение

$$
h=\prod_{i=1}^{r} D\left(v_{i, 1}, \ldots, v_{i, p}, y_{i}\right)=0 \quad\left(\bmod \widetilde{T}^{(3)}\right)
$$

ДокаЗАтельство. Пусть $f \in \widetilde{F}$ - полином и $A \subset\left\{x_{1}, y_{1}, \ldots, x_{n}, y_{n}, \ldots\right\}$. Обозначим через $e(f, A)$ полином, получаемый из $f$ подстановкой единицы вместо каждой буквы из $A$. Легко проверить, что для слова $v \in \widetilde{F}$ и переменной $z_{0} \in \widetilde{F}$ справедлива формула

$$
\left[z_{0}, v\right]=\sum_{z \in v} \operatorname{deg}_{z} v\left[z_{0}, z\right] z^{\left(\operatorname{deg}_{z} v\right)-1} e(v,\{z\}) \quad\left(\bmod \widetilde{T}^{(3)}\right) .
$$

Отсюда следует, что для слова $v \in \widetilde{F}$ такого, что $\operatorname{deg}_{z} v=0(\bmod p)$ при $z \neq z_{0}$, выполнено соотношение $\left[z_{0}, v\right]=0\left(\bmod \widetilde{T}^{(3)}\right)$.

Предположим теперь, что $g_{1}, \ldots, g_{s}$ - однородные полиномы из $\widetilde{F}$ такие, что их образы при факторизации по $\widetilde{T}^{(3)}$ принадлежат центру алгебры $\widetilde{F} / \widetilde{T}^{(3)}$.

Пусть $u \in \widetilde{F}$ - такое слово, что $\operatorname{deg}_{z} u f_{1} \cdots f_{s}=0(\bmod p)$ для любого $z \neq z_{0}$.

Тогда в силу предыдущих замечаний имеем $\left[z_{0}, u g_{1} \cdots g_{s}\right]=0\left(\bmod \widetilde{T}^{(3)}\right)$ и, следовательно,

$$
z_{0} u g_{1} \cdots g_{s}=u g_{1} \cdots g_{s} z_{0}=u z_{0} g_{1} \cdots g_{s} \quad\left(\bmod \widetilde{T}^{(3)}\right) \text {. }
$$

Эти соотношения означают, что справедлива

Лемма 9. Пусть $w$ - слово из $\widetilde{F}$ такое, что полином $w g_{1} \cdots g_{s}$ p-полный, а слово $w^{\prime}$ получается из $ш$ ииклическим сдвигом. Тогда

$$
w g_{1} \cdots g_{s}=w^{\prime} g_{1} \cdots g_{s} \quad(\bmod \widetilde{F}) .
$$

Выясним, какие полиномы алгебры $F$ переходят в элементы центра при факторизации по $\widetilde{T}^{(3)}$. Введем обозначение:

$$
C\left(f_{1}, \ldots, f_{p}, f_{p+1}\right)=\sum_{i=1}^{p} f_{1} \cdots f_{i-1}\left[f_{i}, f_{p+1}\right] f_{p+1}^{p-1} f_{i+1} \cdots f_{p}
$$

Имеем следуюшее соотношение:

$$
\begin{aligned}
& {\left[C\left(x_{1}, \ldots, x_{p}, y_{1}\right), z\right]=\left[\sum_{i=1}^{p} x_{1} \cdots x_{i-1}\left[x_{i}, y_{1}\right] y_{1}^{p-1} x_{i+1} \cdots x_{p}, z\right]} \\
& \quad=\sum_{i=1}^{p} \sum_{\substack{j=1 \\
j \neq i}}^{p}\left[x_{j}, z\right]\left[x_{i}, y_{1}\right] y_{1}^{p-1} e\left(x_{1} \cdots x_{p},\left\{x_{i}, x_{j}\right\}\right) \\
& \quad=\sum_{i=1}^{p} \sum_{j<i}^{p}\left(\left[x_{j}, z\right]\left[x_{i}, y_{1}\right]+\left[x_{i}, z\right]\left[x_{j}, y_{1}\right]\right) y_{1}^{p-1} e\left(x_{1} \cdots x_{p},\left\{x_{i}, x_{j}\right\}\right) \\
& \quad=0\left(\bmod \widetilde{T}^{(3)}\right) .
\end{aligned}
$$


ЛЕмма 10. Полином $h$ представляется по модулю Т-идеала $\widetilde{T}^{(3)}$ как линейная комбиначия полиномов вида

$$
\prod_{i=1}^{l} C\left(v_{i, 1}, u_{i, 2}, \ldots, u_{i, p}, y_{i}\right) \prod_{j=l+1}^{r} D\left(v_{j, 1}, \ldots, v_{j, p}, y_{j}\right)
$$

где для любого $i=1, \ldots, l$ набор $u_{i, 2}, \ldots, u_{i, p}$ есть некоторая перестановка набора $v_{i, 2}, \ldots, v_{i, p}$.

ДокАЗАТЕЛЬСТво проведем индукцией по $l$. Для $l=0$ любое выражение (12) совпадает с $h$, и поэтому наше утверждение верно. Предположим, что для $l=t-1$ утверждение вьполнено: полином $D\left(v_{t, 1}, \ldots, v_{t, p}, y_{t}\right)$ представим в виде линейной комбинации полиномов вида

$$
\begin{aligned}
\xi\left(v_{t, 1}, u_{t, 2}, \ldots, u_{t, p}, y_{t}\right)= & \sum_{s=0}^{p-2} u_{t, p-s+1} \cdots u_{t, p} v_{t, 1} u_{t, 2} \cdots u_{t, p-s-1}\left[u_{t, p-s}, y_{t}\right] y_{t}^{p-1} \\
& +u_{t, 2} \cdots u_{t, p}\left[v_{t, 1}, y_{t}\right] y_{t}^{p-1}
\end{aligned}
$$

где $u_{i, 2}, \ldots, u_{i, p}$ - некоторая перестановка набора $v_{i, 2}, \ldots, v_{i, p}$.

В силу соотношений (6) и (11) справедливо равенство

$$
\begin{array}{r}
\prod_{i=1}^{t-1} C\left(v_{i, 1}, u_{i, 2}, \ldots, u_{i, p}, y_{i}\right) \prod_{j=t}^{r} D\left(v_{j, 1}, \ldots, v_{j, p}, y_{j}\right) \\
=D\left(v_{t, 1}, \ldots, v_{t, p}, y_{t}\right) g=\sum\left\{\xi\left(v_{t, 1}, u_{t, 2}, \ldots, u_{t, p}, y_{t}\right) g:\right. \\
\left.u_{t, 2}, \ldots, u_{t, p}-\text { перестановка набора } v_{t, 2}, \ldots, v_{t, p}\right\},
\end{array}
$$

где

$$
g=\prod_{i=1}^{t-1} C\left(v_{i, 1}, u_{i, 2}, \ldots, u_{i, p}, y_{i}\right) \prod_{j=t+1}^{r} D\left(v_{j, 1}, \ldots, v_{j, p}, y_{j}\right)
$$

Если $w$-какой-нибудь моном полинома $\xi\left(v_{t, 1}, u_{t, 2}, \ldots, u_{t, p}, y_{t}\right)$, где $u_{t, 2}, \ldots, u_{t, p}$ - перестановка набора $v_{t, 2}, \ldots, v_{t, p}$, то полином $w g$-полный. По лемме 4 замена $w$ на циклически сдвинутое слово в полиноме $w g$ дает полином, равный $w g$ по модулю $\widetilde{T}^{(3)}$. Отсюда получаем следуюшие соотношения:

$$
\begin{aligned}
& u_{t, p-s+1} \cdots u_{t, p} v_{t, 1} u_{t, 2} \cdots u_{t, p-s-1}\left[u_{t, p-s}, y_{t}\right] y_{t}^{p-1} \\
& \quad=v_{t, 1} u_{t, 2} \cdots u_{t, p-s-1}\left[u_{t, p-s}, y_{t}\right] y_{t}^{p-1} u_{t, p-s+1} \cdots u_{t, p} g\left(\bmod \widetilde{T}^{(3)}\right)
\end{aligned}
$$

при $s=0, \ldots, p-2$ и

$$
u_{t, 2} \cdots u_{t, p}\left[v_{t, 1}, y_{t}\right] y_{t}^{p-1} g=\left[v_{t, 1}, y_{t}\right] y_{t}^{p-1} u_{t, 2} \cdots u_{t, p} g \quad\left(\bmod \widetilde{T}^{(3)}\right)
$$


В силу этого имеем

$$
\begin{aligned}
& \prod_{i=1}^{t-1} C\left(v_{i, 1}, u_{i, 2}, \ldots, u_{i, p}, y_{i}\right) \prod_{j=t}^{r} D\left(v_{j, 1}, \ldots, v_{j, p}, y_{j}\right) \\
& =\sum\left\{C\left(v_{t, 1}, u_{t, 2}, \ldots, u_{t, p}, y_{t}\right) g:\right. \\
& \left.\quad u_{t, 2}, \ldots, u_{t, p}-\text { перестановка набора } v_{t, 2}, \ldots, v_{t, p}\right\} \\
& =\sum\left\{\prod_{i=1}^{t} C\left(v_{i, 1}, u_{i, 2}, \ldots, u_{i, p}, y_{i}\right) \prod_{j=t+1}^{r} D\left(v_{j, 1}, \ldots, v_{j, p}, y_{j}\right):\right. \\
& \left.u_{t, 2}, \ldots, u_{t, p}-\text { перестановка набора } v_{t, 2}, \ldots, v_{t, p}\right\}\left(\bmod \widetilde{T}^{(3)}\right) .
\end{aligned}
$$

Таким образом, лемма доказана.

Воспользуемся этой леммой при $l=r$. Получаем, что $h$ представляется по модулю $\widetilde{T}^{(3)}$ как линейная комбинация выражений

$$
\prod_{i=1}^{r} C\left(v_{i, 1}, u_{i, 2}, \ldots, u_{i, p}, y_{i}\right)
$$

где для $i=1, \ldots, r$ набор $u_{i, 2}, \ldots, u_{i, p}$ есть перестановка набора $v_{i, 2}, \ldots, v_{i, p}$.

Докажем теперь равенство нулю по модулю $T$-идеала $\widetilde{T}^{(3)}$ всех полиномов (13). Справедливо следующее соотношение:

$$
\begin{aligned}
& C\left(v_{i, 1}, u_{i, 2}, \ldots, u_{i, p}, y_{i}\right) \\
& \quad=\sum_{k=1}^{\infty} a_{i, k}\left[x_{k}, y_{i}\right] x_{k}^{a_{i, k}-1} y_{i}^{p-1} e\left(v_{i, 1} u_{i, 2} \cdots u_{i, p},\left\{x_{k}\right\}\right)\left(\bmod \widetilde{T}^{(3)}\right),
\end{aligned}
$$

где $a_{i, k}=\operatorname{deg}_{x_{k}} v_{i, 1} u_{i, 2} \cdots u_{i, p}$. Тогда имеем

$$
\begin{aligned}
& \prod_{i=1}^{r} C\left(v_{i, 1}, u_{i, 2}, \ldots, u_{i, p}, y_{i}\right)=\sum_{k_{1}, \ldots, k_{r}} a_{1, k_{1}} \cdots a_{r, k_{r}}\left[x_{k_{1}}, y_{1}\right] \cdots\left[x_{k_{r}}, y_{r}\right] \\
& \times x_{k_{1}}^{a_{1, k_{1}}-1} \cdots x_{k_{r}}^{a_{r, k_{r}}-1} y_{1}^{p-1} \cdots y_{r}^{p-1} e\left(g_{0},\left\{x_{k_{1}}, \ldots, x_{k_{r}}\right\}\right) \\
& =\sum_{k_{1}<\cdots<k_{r}} \sum_{\sigma \in S_{r}} a_{1, k_{\sigma(1)}} \cdots a_{r, k_{\sigma(r)}}\left[x_{k_{\sigma(1)}}, y_{1}\right] \cdots\left[x_{k_{\sigma(r)}}, y_{r}\right] \\
& \times x_{k_{\sigma(1)}}^{a_{1, k}{ }^{-1}} \cdots x_{k_{\sigma(r)}}^{a_{r, k}} y_{1}{ }^{p-1} \cdots y_{r}^{p-1} e\left(g_{0},\left\{x_{k_{1}}, \ldots, x_{k_{r}}\right\}\right) \\
& =\sum_{k_{1}<\cdots<k_{r}}\left(\sum_{\sigma \in S_{r}} \operatorname{sgn}(\sigma) a_{1, k_{\sigma(1)}} \cdots a_{r, k_{\sigma(r)}}\right)\left[x_{k_{1}}, y_{1}\right] \cdots\left[x_{k_{r}}, y_{r}\right] \\
& \times x_{k_{1}}^{a_{1, k_{1}}-1} \cdots x_{k_{r}}^{a_{r, k_{r}}-1} y_{1}^{p-1} \cdots y_{r}^{p-1} e\left(g_{0},\left\{x_{k_{1}}, \ldots, x_{k_{r}}\right\}\right)\left(\bmod \widetilde{T}^{(3)}\right),
\end{aligned}
$$

где $g_{0}=\prod_{i=1}^{r} v_{i, 1} u_{i, 2} \cdots u_{i, p}$. Выражение $\sum_{\sigma \in S_{r}} \operatorname{sgn}(\sigma) a_{1, k_{\sigma(1)}} \cdots a_{r, k_{\sigma(r)}}$ есть определитель матрицы $M_{k_{1}}, \ldots, k_{r}$, у которой на пересечении $i$-й строки и $j$-го столбца стоит элемент $a_{i, k_{j}}$. По условию

$$
\sum_{i=1}^{r} a_{i, k_{j}}=\sum_{i=1}^{r} \operatorname{deg}_{x_{k_{j}}} v_{i, 1} u_{i, 2} \cdots u_{i, p}=\operatorname{deg}_{x_{k_{j}}} g_{0}=0 \quad(\bmod p)
$$


для любого $j=1, \ldots, r$. Следовательно, если рассматривать матрицу $M_{k_{1}, \ldots, k_{r}}$ как матрицу над полем $\mathbb{Z}_{p}$, то ее строки линейно зависимы (их сумма равна нулю по модулю $p$ ). Поэтому ее определитель равен нулю по модулю $p$. Это означает, чTO

$$
\sum_{\sigma \in S_{r}} \operatorname{sgn}(\sigma) a_{1, k_{\sigma(1)}} \cdots a_{r, k_{\sigma(r)}}=0 \quad(\bmod p) .
$$

Отсюда $h=0(\bmod \widetilde{F})$. Лемма 8 доказана.

Имеет место

ЛЕмМа 11. При $р>2$ вьиполняется $\varphi_{m} \notin T^{(3)}$.

ДоКАЗАТЕЛЬСТво. Подставляя в полином $\varphi_{m}$ вместо $x_{i}$ полином $1+x_{i}$ при $i=1, \ldots, m$ и выделяя затем полилинейное однородное слагаемое, зависяшее от всех $\left\{x_{1}, \ldots, x_{m}\right\}$, получаем полином $\left[x_{1}, x_{2}\right] \cdots\left[x_{m-1}, x_{m}\right]$. Если предположить, что $\varphi_{m} \in T^{(3)}$, то в силу унитарной замкнутости $T$-идеала $T^{(3)}$ получим, что

$$
\left[x_{1}, x_{2}\right] \cdots\left[x_{m-1}, x_{m}\right] \in T^{(3)} .
$$

В алгебре Грассмана $G=K\left\langle e_{1}, \ldots, e_{n}, \ldots\right\rangle /\left\{e_{i} e_{j}+e_{j} e_{i}: i, j \in \mathbb{N}\right\}$, как известно, выполняется тождество $[[x, y], z]=0$. С другой стороны, в алгебре $G$ выполняется $\left[e_{1}, e_{2}\right] \cdots\left[e_{m-1}, e_{m}\right]=2^{m / 2} e_{1} \cdots e_{m} \neq 0$, что показывает несправедливость соотношения (14). Таким образом, $\varphi_{m} \notin T^{(3)}$ при $p>2$ (впрочем, и при $p=2$ ). Лемма доказана.

ЛЕмма 12. Полином $\varphi_{m} x_{m+1}^{p} \cdots x_{m^{\prime}}^{p}$ не мохет быть представлен по модулю T-идеала $T^{(3)}$ в виде линейной комбинации однородных полиномов вида $f x_{k_{1}}^{p} \cdots x_{k_{t}}^{p}$, где $\operatorname{deg}_{x_{k}} f x_{k_{1}}^{p} \cdots x_{k_{t}}^{p}=\operatorname{deg}_{x_{k}} \varphi_{m} x_{m+1}^{p} \cdots x_{m^{\prime}}^{p}$ для любой переменной $x_{k} \in \widetilde{F}$ имножество $\left\{k_{1}, \ldots, k_{t}\right\}$ не является подмножсеством множества $\left\{m+1, \ldots, m^{\prime}\right\}$.

ДокАЗАтЕльство. Подставляя вместо переменных $x_{m+1}, \ldots, x_{m^{\prime}}$ единицу и учитывая унитарную замкнутость $T$-идеала $T^{(3)}$, получаем, что лемму достаточно доказать для $m^{\prime}=m$. Проведем индукцию по $m$. Для $m=0$ утверждение очевидно. Пусть утверждение верно для $m=n-2$. Докажем его для $m=n$. Предположим, что это не так. Тогда

$$
\begin{aligned}
\varphi_{m}= & \sum_{k=1}^{k_{0}} \alpha_{k} x_{i(1, k)}^{p-1}\left[x_{i(1, k)}, x_{i(2, k)}\right] x_{i(2, k)}^{p-1} \cdots x_{i\left(s_{k}-1, k\right)}^{p-1} \\
& \times\left[x_{i\left(s_{k}-1, k\right)}, x_{i\left(s_{k}, k\right)}\right] x_{i\left(s_{k}, k\right)}^{p-1} x_{j(1, k)}^{p} \cdots x_{j\left(r_{k}, k\right)}^{p}\left(\bmod T^{(3)}\right) .
\end{aligned}
$$

В силу леммы 11 можно считать, что $k_{0} \geqslant 1$ и $\alpha_{k} \neq 0, r_{k} \geqslant 1$ для $k=1, \ldots, k_{0}$.

Считаем также, что любой член суммы из правой части формулы (15) имеет ту же степень по любой переменной $x_{k} \in F$, что и полином $\varphi_{m}$, и при этом для $k \neq l$ вьполнено $\left\{j(1, k), \ldots, j\left(r_{k}, k\right)\right\} \neq\left\{j(1, l), \ldots, j\left(r_{l}, l\right)\right\}$. Подставляя вместо переменных $x_{j_{1,1}}, \ldots, x_{j_{r_{1}, 1}}$ единицу, получаем противоречие для некоторого $m \leqslant$ $n-2$. Лемма доказана. 
Если предположить, что при $q \geqslant 1$ и выполнении вышеупомянутых условий на $v_{1,1}, \ldots, v_{2 q, p}$ справедливо

$$
\psi_{2 q}\left(v_{1,1}, \ldots, v_{2 q, p}, v_{2 q+1}, \ldots, v_{m}\right) \neq 0 \quad\left(\bmod T^{(3)}\right),
$$

TO

$$
\begin{aligned}
& \psi_{2 q}\left(v_{1,1}, \ldots, v_{2 q, p}, v_{2 q+1}, \ldots, v_{m}\right) \\
& =\sum_{k=1}^{k_{0}} \alpha_{k} x_{i(1, k)}^{p-1}\left[x_{i(1, k)}, x_{i(2, k)}\right] x_{i(2, k)}^{p-1} \cdots x_{i\left(s_{k}-1, k\right)}^{p-1} \\
& \quad \times\left[x_{i\left(s_{k}-1, k\right)}, x_{i\left(s_{k}, k\right)}\right] x_{i\left(s_{k}, k\right)}^{p-1} x_{j(1, k)}^{p} \cdots x_{j\left(r_{k}, k\right)}^{p}\left(\bmod T^{(3)}\right),
\end{aligned}
$$

где опять $k_{0} \geqslant 1, \alpha_{k} \neq 0, r_{k} \geqslant 1$ для $k=1, \ldots, k_{0}$, любой член суммы из правой части данной формулы имеет ту же степень по любой переменной $x_{k} \in F$, что и полином $\psi_{2 q}\left(v_{1,1}, \ldots, v_{2 q, p}, v_{2 q+1}, \ldots, v_{m}\right)$, и при этом для $k \neq l$ выполнено $\left\{j(1, k), \ldots, j\left(r_{k}, k\right)\right\} \neq\left\{j(1, l), \ldots, j\left(r_{l}, l\right)\right\}$.

Умножая $\psi_{2 q}\left(v_{1,1}, \ldots, v_{2 q, p}, v_{2 q+1}, \ldots, v_{m}\right)$ на

$$
y_{1}^{p-1}\left[y_{1}, y_{2}\right] y_{2}^{p-1} \cdots y_{m-1}^{p-1}\left[y_{m-1}, y_{m}\right] y_{m}^{p-1},
$$

подставляя единицу вместо $x_{j(1,1)}, \ldots, x_{j\left(r_{1}, 1\right)}$ и учитывая лемму 8, приходим к противоречию с леммой 12 .

Аналогично при $q \geqslant 1$ и выполнении вышеупомянутых условий на $v_{1,1}, \ldots$, $v_{2 q-1, p}$ получаем

$$
\psi_{2 q-1}\left(v_{1,1}, \ldots, v_{2 q-1, p}, v_{2 q}, \ldots, v_{m}\right)=0 \quad\left(\bmod T^{(3)}\right) .
$$

Таким образом, можно считать, что $T$-пространство, порожденное полиномом $\varphi_{m}$ и $T$-идеалом $T^{(3)}$, натянуто на полиномы вида

$$
v_{1}^{p-1}\left[v_{1}, v_{2}\right] v_{2}^{p-1} \cdots v_{m-1}^{p-1}\left[v_{m-1}, v_{m}\right] v_{m}^{p-1}
$$

где $v_{1}, \ldots, v_{m}$ - некоторые слова из $F$.

Считаем теперь, что $p>2$. Тогда имеем

$$
\left(x_{1} x_{2}\right)^{p}=x_{1}^{p} x_{2}^{p}+\frac{p(p-1)}{2}\left[x_{2}, x_{1}\right] x_{1}^{p-1} x_{2}^{p-1}=x_{1}^{p} x_{2}^{p} \quad\left(\bmod T^{(3)}\right),
$$

так как число $p(p-1) / 2$ делится на $p$ при $p>2$.

Если существует $i=1, \ldots, m$ такое, что слово $v_{i}$ не однобуквенно, то так как выполняется соотношение

$$
\left[y_{1}, v_{i}\right] v_{i}^{p-1}=\sum_{z \in v_{i}} \operatorname{deg}_{z} v_{i}\left[y_{1}, z\right] z^{\left(\operatorname{deg}_{z} v_{i}\right)-1} e\left(v_{i}^{p},\{z\}\right) \quad\left(\bmod \widetilde{T}^{(3)}\right)
$$

в силу (17) получаем, что (16) представляется в виде линейной комбинации полиномов вида $x_{i}^{p} f$. Поэтому предположение о том, что полином $\varphi_{m+2}$ принадлежит $T$-пространству, порожденному полиномами $\varphi_{2}, \ldots, \varphi_{m}$ и $T$-идеалом $T^{(3)}$, противоречит лемме 12 . Таким образом, получаем следующую теорему. 
ТЕОРема 4. T-пространство алгебры $F$, поро сденное полиномами $\varphi_{2}, \ldots$, $\varphi_{m}, \ldots$, при $p>2$ не является конечно базируемым по модулю тохдества $[[x, y], z]=0$.

ЗАМЕЧАНИЕ. Как легко видеть, при $p>2$ выполняется соотношение

$$
\begin{aligned}
& x_{2} x_{1}^{p-1}+x_{1} x_{2} x_{1}^{p-2}+\cdots+x_{1}^{p-1} x_{2} \\
& \quad=\frac{p(p-1)}{2}\left[x_{2}, x_{1}\right] x_{1}^{p-1}+p x_{2} x_{1}^{p-1}=0\left(\bmod T^{(3)}\right) .
\end{aligned}
$$

Линеаризуя это соотношение, получаем

$$
\sum_{\sigma \in S_{p}} x_{\sigma(1)} \cdots x_{\sigma(p)}=0 \quad\left(\bmod T^{(3)}\right) \text {. }
$$

Если предположить, что $\varphi_{m}$ принадлежит идеалу, порожденному $T$-идеалом $T^{(3)}$ и полиномами $f^{p}$, где $f$ - полином без свободного члена, то получим, что полином $\left[x_{1}, x_{2}\right] \cdots\left[x_{m-1}, x_{m}\right]$ принадлежит $T$-идеалу, порожденному полиномами $\left[\left[x_{1}, x_{2}\right], x_{3}\right]$ и $\sum_{\sigma \in S_{p}} x_{\sigma(1)} \cdots x_{\sigma(p)}$. В силу соотношения (18) получаем тогда $\left[x_{1}, x_{2}\right] \cdots\left[x_{m-1}, x_{m}\right] \in T^{(3)}$, что неверно. Это означает, что справедлива

ЛЕмма 13. Т-пространство, порожденное полиномами $\varphi_{2}, \ldots, \varphi_{m}, \ldots$, при $p>2$ не является конечно базируемым по модулю идеала, порожденного $T$-идеалом $T^{(3)}$ и полиномами $f^{p}=0$, где $f-$ полином без свободного члена.

Пусть $Q^{\prime}(f, g)=f^{p-1} g f g^{p-1}$ и для четного $m$ положим

$$
\varphi_{m}^{\prime}=Q^{\prime}\left(x_{1}, x_{2}\right) \cdots Q^{\prime}\left(x_{m-1}, x_{m}\right)
$$

\section{Справедлива}

ТеОРема 5. Т-пространство, порохсенное полиномами $\varphi_{2}^{\prime}, \ldots, \varphi_{m}^{\prime}, \ldots$, при $р>2$ не является конечно базируемым по модулю идеала, порожденного T-идеалом $T^{(3)}$ и полиномами $f^{p}=0$, где $f$ - полином без свободного члена.

ДокАЗАТЕЛЬство. Через $L_{m}$ обозначим $T$-пространство, порожденное полиномами $1, \varphi_{2}, \ldots, \varphi_{m}, f^{p}$, где $f$ - полином без свободного члена, и $T$-идеалом $T^{(3)}$. Считаем, что $\left(n_{2}, l_{2}\right)>\left(n_{1}, l_{1}\right)$, где $n_{2}, l_{2}, n_{1}, l_{1}$ - неотрищательные целые числа, если пара $\left(n_{2}, l_{2}\right)$ лексикографически больше пары $\left(n_{1}, l_{1}\right)$.

Докажем, что при $l \leqslant n \leqslant m / 2$ выражение

$$
Q^{\prime}\left(f_{1}, g_{1}\right) \cdots Q^{\prime}\left(f_{l}, g_{l}\right) Q\left(f_{l+1}, g_{l+1}\right) \cdots Q\left(f_{n}, g_{n}\right)
$$

принадлежит $L_{m}$.

Проведем индукцию по паре $(n, l)$.

Пусть $l \geqslant 1$. Пусть также $f_{l}=\bar{f}_{l}+\alpha$ и $g_{l}=\bar{g}_{l}+\beta$, где $\alpha, \beta \in K$, а $\bar{f}_{l}$ и $\bar{g}_{l}-$ полиномы без свободного члена.

Тогда

$$
f_{l}^{p} g_{l}^{p}=\left(\bar{f}_{l}^{p}+\alpha^{p}\right)\left(\bar{g}_{l}^{p}+\beta^{p}\right)=\alpha^{p} \beta^{p} \quad\left(\bmod L_{m}\right)
$$


Отсюда

$Q^{\prime}\left(f_{l}, g_{l}\right)=f_{l}^{p-1} g_{l} f_{l} g_{l}^{p-1}-\left(f_{l}^{p} g_{l}^{p}-\alpha^{p} \beta^{p}\right)=-Q\left(f_{l}, g_{l}\right)+\alpha^{p} \beta^{p} \quad\left(\bmod L_{m}\right)$

Следовательно,

$$
\begin{aligned}
& Q^{\prime}\left(f_{1}, g_{1}\right) \cdots Q^{\prime}\left(f_{l}, g_{l}\right) Q\left(f_{l+1}, g_{l+1}\right) \cdots Q\left(f_{n}, g_{n}\right) \\
& \quad=-Q^{\prime}\left(f_{1}, g_{1}\right) \cdots Q^{\prime}\left(f_{l-1}, g_{l-1}\right) Q\left(f_{l}, g_{l}\right) \cdots Q\left(f_{n}, g_{n}\right) \\
& \quad+\alpha^{p} \beta^{p} Q^{\prime}\left(f_{1}, g_{1}\right) \cdots Q^{\prime}\left(f_{l-1}, g_{l-1}\right) Q\left(f_{l+1}, g_{l+1}\right) \cdots Q\left(f_{n}, g_{n}\right)\left(\bmod L_{m}\right) .
\end{aligned}
$$

Два последних слагаемых принадлежат $L_{m}$ по предположению индукции. Следовательно,

$$
Q^{\prime}\left(f_{1}, g_{1}\right) \cdots Q^{\prime}\left(f_{l}, g_{l}\right) Q\left(f_{l+1}, g_{l+1}\right) \cdots Q\left(f_{n}, g_{n}\right)=L_{m}
$$

Если же $l=0$, то выражение (19) принадлежит $L_{m}$ по определению. По лемме $13 \varphi_{m+2} \notin L_{m}$. С другой стороны, $\varphi_{m+2}=\varphi_{m+2}^{\prime}\left(\bmod L_{m}\right)$. Поэтому $\varphi_{m+2} \notin L_{m}$.

Теорема доказана.

\section{§5. Конечная базируемость некоторых $T$-пространств}

Пусть $R=\Phi\langle X\rangle$, где $\Phi$ - коммутативное кольцо с единицей, $X=\left\{x_{1}, \ldots, x_{k}, \ldots\right\}$. Для $f_{1}, \ldots, f_{d} \in R$ положим

$$
S_{d}\left(f_{1}, \ldots, f_{d}\right)=\sum_{\sigma \in S(d)} f_{\sigma(1)} \cdots f_{\sigma(d)} .
$$

Пусть $h_{n}=S_{d}\left(x_{1}, \ldots, x_{d}\right) \cdots S_{d}\left(x_{(n-1) d+1}, \ldots, x_{n d}\right)$.

Обозначим через $R_{n} \quad T$-пространство алгебры $R$, порожденное полиномами $h_{1}, \ldots, h_{n}$. Докажем, что $R_{d+1}=R_{d}$.

Для удобства изложения через $P$ обозначим множество полилинейных многочленов алгебры $R(R$ не является подмодулем модуля $\Phi R)$. Для $f \in P$ положим vr $f$ равным множеству переменных, от которых зависит $f$.

ЛЕмма 14. Пусть $h=\sum_{i=1}^{k} g_{i} f g_{i}^{\prime} \in L \cap P$, әде $L-T$-пространство алгебpъ R. Тогда $\sum_{i=1}^{k} g_{i}\left[f, x_{k}\right] g_{i}^{\prime} \in L$.

ДокАЗАТЕльство. Пусть $\operatorname{vr} f=\left\{x_{i(1)}, \ldots, x_{i(m)}\right\}$. Через $f_{i}^{\prime}$ обозначим полином, получаюшийся из $f$ подстановкой: $x_{i(l)} \rightarrow x_{i(l)}$ при $l \neq j$ и $x_{i(j)} \rightarrow x_{i(j)} x_{k}$, а через $f_{j}^{\prime \prime}$ - полином, получаюшийся из $f$ подстановкой: $x_{i(l)} \rightarrow x_{i(l)}$ при $l \neq j$ и $x_{i(j)} \rightarrow x_{k} x_{i(j)}$.

Тогда $L \ni \sum_{j=1}^{m} \sum_{i=1}^{s} g_{i} f_{j}^{\prime} g_{i}^{\prime}-\sum_{j=1}^{m} \sum_{i=1}^{s} g_{i} f_{j}^{\prime \prime} g_{i}^{\prime}=\sum_{j=1}^{m} \sum_{i=1}^{s} g_{i}\left(f_{i}^{\prime}-f_{i}^{\prime \prime}\right) g_{i}^{\prime}=$ $\sum_{i=1}^{s} g_{i}\left[f, x_{k}\right] g_{i}^{\prime}$.

Лемма доказана.

СлЕДСТВИЕ ИЗ лЕммы 14. Пусть $L-T$-пространство алгебры $R$. Тогда

1) $g \in L \Rightarrow\left[g, x_{k}\right] \in L$,

2) $\sum_{i=1}^{s} g_{i} x_{k} f g_{i}^{\prime} \in L \cap P \Rightarrow \sum_{i=1}^{k} g_{i} x_{k} f x_{l} g_{i}^{\prime} \in L$. 
ДокАЗАТЕЛЬство. Первое свойство легко следует из правила дифференцирования Лейбница. Докажем второе. Имеем $\sum_{i=1}^{k} g_{i} x_{l} x_{k} f g_{i}^{\prime} \in L$. По лемме 14 имеем также $\sum_{i=1}^{k} g_{i}\left[x_{k} f, x_{l}\right] g_{i}^{\prime} \in L$. Суммируя эти выражения, получаем $\sum_{i=1}^{k} g_{i} x_{k} f x_{l} g_{l}^{\prime} \in L$.

Введем обозначение: $S_{d+1}^{\prime}\left(f_{1}, \ldots, f_{d}, g\right)=\sum_{i=1}^{d} S_{d}\left(f_{1}, \ldots, g f_{i}, \ldots, f_{d}\right)$.

Тогда, как легко проверить,

$$
S_{d+1}^{\prime}\left(f_{1}, \ldots, f_{d}, g\right)=\sum_{i=1}^{d} S_{d}\left(f_{1}, \ldots, f_{i-1}, g, f_{i+1}, \ldots, f_{d}\right) f_{i}
$$

Пусть $u_{2}, \ldots, u_{d} \in R_{1}$ - полиномы, не содержащие переменные $x_{1}, \ldots, x_{d+1}$.

Построим последовательность многочленов $\left\{f_{i}: i=1, \ldots, d\right\}$ по следующему правилу:

$$
\begin{aligned}
f_{1}\left(x_{1}, \ldots, x_{d+1}\right)= & S_{d+1}^{\prime}\left(x_{1}, \ldots, x_{d+1}\right) \\
f_{k+1}\left(x_{1}, \ldots, x_{d+1}\right)= & u_{k+1} f_{k}\left(x_{1}, \ldots, x_{d+1}\right) \\
& -f_{k}\left(x_{1}, \ldots, x_{k-1}, x_{k+1} u_{k+1}, x_{k+2}, \ldots, x_{d+1}\right),
\end{aligned}
$$

где $k=1, \ldots, d-1$

Лемма 15. Справедливо равенство

$$
\begin{aligned}
& f_{k}\left(x_{1}, \ldots, x_{d+1}\right)=\sum_{j_{2}, \ldots, j_{k} \in\{0,1\}}(-1)^{j_{2}+\cdots+j_{k}} u_{k}^{1-j_{k}} \cdots u_{2}^{1-j_{2}} \\
& \quad \times S_{d}\left(x_{2} u_{2}^{j_{2}}, \ldots, x_{k} u_{k}^{j_{k}}, x_{k+1}, \ldots, x_{d+1}\right) x_{1}+\sum_{i=k+1}^{d} g_{i} x_{i}\left(\bmod R_{1}\right),
\end{aligned}
$$

где $g_{k+1}, \ldots, g_{d}$ - некоторые многочлены такие, что полином $g_{i}$ не содержит переменной $x_{i}$.

ДокАЗАТЕльство. Проведем индукцию по $k$. Для $f_{1}$ утверждение справедливо по формуле (20). Пусть $k<d$ и для $k$ формула (22) выполнена. Имеем в силу (21)

$$
\begin{aligned}
f_{k+1}\left(x_{1}, \ldots, x_{d+1}\right) & \sum_{j_{2}, \ldots, j_{k} \in\{0,1\}}(-1)^{j_{2}+\cdots+j_{k}} u_{k+1} u_{k}^{1-j_{k}} \cdots u_{2}^{1-j_{2}} \\
& \quad \times S_{d}\left(x_{2} u_{2}^{j_{2}}, \ldots, x_{k} u_{k}^{j_{k}}, x_{k+1}, \ldots, x_{d+1}\right) x_{1} \\
& +\sum_{j_{2}, \ldots, j_{k} \in\{0,1\}}(-1)^{j_{2}+\cdots+j_{k}+1} u_{k}^{1-j_{k}} \cdots u_{2}^{1-j_{2}} \\
& \quad \times S_{d}\left(x_{2} u_{2}^{j_{2}}, \ldots, x_{k} u_{k}^{j_{k}}, x_{k+1} u_{k+1}, \ldots, x_{d+1}\right) x_{1}+\left[u_{k+1}, g_{k+1} x_{k+1}\right] \\
& +\sum_{i=k+2}^{d} g_{i}^{\prime} x_{i}=\sum_{j_{2}, \ldots, j_{k+1} \in\{0,1\}}(-1)^{j_{2}+\cdots+j_{k}} u_{k+1}^{1-j_{k+1}} u_{k}^{1-j_{k}} \ldots u_{2}^{1-j_{2}} \\
& \quad \times S_{d}\left(x_{2} u_{2}^{j_{2}}, \ldots, x_{k} u_{k}^{j_{k}}, x_{k+1} u_{k+1}^{j_{k+1}}, x_{k+2}, \ldots, x_{d+1}\right) x_{1} \\
& +\sum_{i=k+2}^{d} g_{i}^{\prime} x_{i}\left(\bmod R_{1}\right) .
\end{aligned}
$$


Лемма доказана.

При $k=d$ формула (22) принимает вид:

$$
\begin{aligned}
& f_{d}\left(x_{1}, \ldots, x_{d+1}\right) \\
& \quad=\sum_{j_{2}, \ldots, j_{d} \in\{0,1\}}(-1)^{j_{1}+\cdots+j_{d}} u_{d}^{1-j_{d}} \cdots u_{2}^{1-j_{2}} S_{d}\left(x_{2} u_{2}^{j_{2}}, \ldots, x_{d} u_{d}^{j_{d}}, x_{d+1}\right) x_{1} .
\end{aligned}
$$

Из этой формулы следует, что $f_{d} \in R_{d}$.

ЛЕМма 16. $R_{d+1}=R_{d}$.

ДокАЗАТЕЛьство. Подставим вместо $x_{1}$ элемент $u_{d+1}$ из $R_{1}$. Тогда имеем соотношение

$$
\begin{aligned}
& u_{d} \cdots u_{2} S_{p}\left(x_{2}, \ldots, x_{d}, x_{d+1}\right) u_{d+1} \\
& +\sum_{\substack{j_{2}, \ldots, j_{d} \in\{0,1\} \\
\exists i: j_{i} \neq 0}}(-1)^{j_{2}+\cdots+j_{d}} u_{d}^{1-j_{d}} \cdots u_{2}^{1-j_{2}} S_{d}\left(x_{2} u_{2}^{j_{2}}, \ldots, x_{d} u_{d}^{j_{d}}, x_{d+1}\right) u_{d+1} \in R_{d} .
\end{aligned}
$$

Легко видеть, что выражение, стояшее под знаком суммы в последнем соотношении, принадлежит $R_{d}$. Тогда $u_{d} \cdots u_{2} S_{d}\left(x_{2}, \ldots, x_{d}, x_{d+1}\right) u_{d+1} \in R_{d}$. В силу произвольности выбора полиномов $u_{2}, \ldots, u_{d}, u_{d+1}$ получим $R_{d+1}=R_{d}$. Лемма доказана.

Пусть теперь $K$ - поле характеристики $p>2, F=K\left\langle x_{1}, \ldots, x_{n}, \ldots\right\rangle, L_{n}$ есть $T$-пространство алгебры $F$, порожденное многочленами $\left\{x_{1}^{p} \cdots x_{i}^{p}: i=1, \ldots, n\right\}$.

Докажем, что $L_{p+1}=L_{p}$. Пусть $c=(p+1) / 2$. По доказанному ранее $R_{p+1}=R_{p}$, в частности в случае $\Phi=\mathbb{Z}$. Следовательно,

$$
S_{p}\left(x_{1,1}, \ldots, x_{1, p}\right) \cdots S_{p}\left(x_{p+1,1}, \ldots, x_{p+1, p}\right) \in L_{p},
$$

где $x_{i, j}$ - некоторые буквы алгебры $F$.

Справедлива

ЛЕмМа 17. При $k=0, \ldots, c$ имеем

$$
S_{p}\left(x_{1,1}, \ldots, x_{1, p}\right) \cdots S_{p}\left(x_{2(c-k), 1}, \ldots, x_{2(c-k), p}\right) z_{1}^{p} \cdots z_{2 k}^{p} \in L_{p}
$$

где $x_{i, j}, z_{i}$ - некоторие буквы.

ДокАЗАТЕЛЬСтво. Проведем индукцию по $k$. Для $k=0$ утверждение справедливо в силу (23). Пусть $k \leqslant 1$, тогда по предположению индукции имеем

$$
f S_{p}\left(x_{1}, \ldots, x_{p}\right) S_{p}\left(y_{1}, \ldots, y_{p}\right) z_{3}^{p} \cdots z_{2 k}^{p} \in L_{p}
$$

где $f=S_{p}\left(x_{1,1}, \ldots, x_{1, p}\right) \cdots S_{p}\left(x_{2(c-k), 1}, \ldots, x_{2(c-k), p}\right)$.

Но $f\left(z_{1}+z_{2}\right)^{p} z_{3}^{p} \cdots z_{2 k}^{p} \in L_{p}$. Считая, что переменные $z_{1}$ и $z_{2}$ не входят в полиномы $f$ и $z_{3}^{p} \cdots z_{2 k}^{p}$, выделим из многочлена $f\left(z_{1}+z_{2}\right)^{p} z_{3}^{p} \cdots z_{2 k}^{p}$ однородную компоненту $h$ степени $p$ по переменным $z_{1}$ и $z_{2}$. Тогда $h \in L_{p}$. 
Пусть

$$
g=\sum_{i=1}^{p-1} \frac{1}{(i !(p-i) !)^{2}} f S_{p}(\underbrace{z_{1}, \ldots, z_{1}}_{i \text { раз }}, z_{2}, \ldots, z_{2}) S_{p}(\underbrace{z_{1}, \ldots, z_{1}}_{p-i \text { раз }}, z_{2}, \ldots, z_{2}) z_{3}^{p} \cdots z_{2 k}^{p} .
$$

Заметим, что $i !(p-i) ! \neq 0$ и деление корректно. Имеем $f\left(z_{1}^{p} z_{2}^{p}+z_{2}^{p} z_{1}^{p}\right) z_{3}^{p} \cdots z_{2 k}^{p}$ $=h-g \in L_{p}$. По п. 1) следствия из леммы 14 имеем $f\left[z_{1}^{p}, z_{2}^{p}\right] z_{3}^{p} \cdots z_{2 k}^{p} \in L_{p}$. Следовательно, имеем $2 f z_{1}^{p} z_{2}^{p} z_{3}^{p} \cdots z_{2 k}^{p} \in L_{p}$. Так как $p>2, f z_{1}^{p} z_{2}^{p} z_{3}^{p} \cdots z_{2 k}^{p} \in L_{p}$. Лемма доказана.

При $k=c$ утверждение леммы принимает вид $z_{1}^{p} \cdots z_{p+1}^{p} \in L_{p}$, откуда $L_{p+1}=L_{p}$

Таким образом, доказана

TEOpema 6. $L_{p+1}=L_{p}$.

\section{Список литературы}

1. Schpecht W. Gesetze in Ringen // Math. Z. 1950. V. 52. № 5. P. 557-589.

2. Кемер А.P. Конечная базируемость тождеств ассоциативных алгебр // Алгебра и логика. 1987. Т. 5. С. 597-641.

3. Кемер А.Р. Тождества конечно порожденных алгебр над бесконечньп полем // Изв. АН СССР. Сер. матем. 1990. Т. 54. № 4. С. 726-753.

4. Гришин А.В. О конечной базируемости абстрактных $T$-пространств // Фундамент. и прикл. матем. 1995. Т. 1. № 3. С. 669-700.

5. Гришин A.B. Бесконечно базируемое $T$-пространство над полем характеристики 2 // Международная конференция по алгебре и анализу, посвященная 100 -летию со дня рождения Н. Г. Чеботарева. Тезисы докладов. Казань, 5-11 июня 1994 г. С. 29.

Московский государственный

Поступила в редакцию университет им. М.В. Ломоносова 